\title{
Sleep Diary
}

National Cancer Institute

\section{Source}

National Cancer Institute. Sleep Diary. NCI Thesaurus. Code C159581.

A record of an individual's experiences and observations about their sleeping. This usually includes: time going to bed and time awakening; times awake during the night; and subjective opinion of sleep quality, but can also include information about sleep preparations: time and amount of caffeine or alcohol consumed; food and drink consumed; medications taken; exercise during the day and pre-sleep activities; and emotional or stress status. 